\title{
THE HELIUM-TO-HYDROGEN RATIO OF STARS IN YOUNG CLUSTERS AND ASSOCIATIONS
}

\author{
P. E. NISSEN
}

Institute of Astronomy, University of Aarhus, Denmark

\begin{abstract}
A photoelectric narrow-band index, I(4026), of the He I $\lambda 4026$ line has been observed with an echelle spectrometer for B-type stars in young clusters and associations.

Model-atmosphere computations of the Strömgren $\left[c_{1}\right]$ index, the $\mathrm{H} \beta$ index, and $\mathrm{I}(4026)$ as functions of $T_{\text {eff, }} g$ and the helium-to-hydrogen ratio have been used to derive these atmospheric parameters from the observed indices for each star.

For the $\mathrm{OriOBI}_{b}$, LacOBI and Sco-Cen associations 11 out of 48 stars in the temperature range from 14000 to $20000 \mathrm{~K}$ are found to be helium poor in the sense that the derived heliumto-hydrogen ratios lie in the range from 0.05 down to very low values. In the temperature range from 20000 to $30000 \mathrm{~K}$ no helium poor stars are found, but two stars in OriOBI $_{b}$ are helium rich. Both of these stars show rather strong variations of the $\mathrm{I}(4026)$ index.

All stars in $h+\chi$ Per and CepOBIII are apparently helium deficient compared to nearby field stars.

In a review paper on photoelectric narrow-band photometry B. Strömgren (1966) proposed to observe an index of one of the helium lines in the spectra of B-type stars in order to determine the helium abundances of a large sample of stars in the Galaxy. Following this proposal I have observed an index, I(4026), of the strength of the He I $\lambda 4026$ absorption line for several hundred B-type stars.
\end{abstract}

The $I(4026)$ index is defined as:

$$
I(4026)=2.5 \log \frac{F_{14}(4026)}{F_{6}(4016)+F_{6}(4036)},
$$

where $F_{14}(4026)$ is the flux of the star in a $14 \AA$ wide spectral band centred on He I $\lambda 4026$, and $F_{6}(4016)+F_{6}(4036)$ is the total flux of two $6 \AA$ wide 'continuum' bands at $4016 \AA$ and $4036 \AA$ respectively. The bands are defined by exit slots in the spectrum formed by an echelle spectrometer with a linear dispersion of $1.3 \AA \mathrm{mm}^{-1}$ at $4026 \AA$. The intensity of the light passing the slots is measured with pulse-counting technique.

In an earlier publication (Nissen, 1974) it was described how model-atmosphere computations of the Strömgren $\left[c_{1}\right]$ index, the $\mathrm{H} \beta$ index, and $I(4026)$ as functions of $T_{\text {eff }}, g$ and the helium-to-hydrogen ratio, $\epsilon(\mathrm{He})=N_{\mathrm{He}} / N_{\mathrm{H}}$, could be used to derive these stellar atmospheric parameters for each observed star. It was found that the mean helium abundance of field stars, that lie within $500 \mathrm{pc}$ from the sun, was about $\epsilon(\mathrm{He})=0.10$. The rms scatter of $\epsilon(\mathrm{He})$ was found to be smaller than 0.01 . Furthermore stars in the rather distant cluster, NGC 6231 , were found to have about the same mean helium abundance as the field stars.

In the present paper I want to describe some new results, that are based on observations of $I(4026)$ recently obtained with the ESO $100 \mathrm{~cm}$ telescope at La Silla, Chile, and the $193 \mathrm{~cm}$ telescope at Observatoire de Haute Provence, France. The observations 
include the Sco-Cen, OriOBI $b$ and LacOBI associations, for which the whole range of $\mathrm{B}$ spectral types was covered, and $h+\chi$ Per and CepOBIII, for which the observations were confined to the spectral range B0-B3.

For Sco-Cen, OriOBI $b$ and $\mathrm{LacOBI} 11$ out of 48 stars in the effective temperature range from 14000 to $20000 \mathrm{~K}$ are found to be helium poor in the sense that the derived helium abundances lie in the range from $\epsilon(\mathrm{He})=0.05$ down to very low values. In Sco-Cen these stars are HD 142884, 144334, 151346, 142990, 143699, and 146001, of which the first three have been classified as weak helium-line stars by Garrison (1967). In $\mathrm{OriOBI}_{b}$ the helium-poor stars are HD 36046, 36526, 37525, 37642, and finally one star in LacOBI, HD 213918, was found to be helium poor. For all these stars there is a rather large inconsistency between the $\left[c_{1}\right]$ index and the spectral type, but I remark that for the stars in $\mathrm{OriOBI}_{b}$ and LacOBI I have been able to find HD types only.

In the effective temperature range from 20000 to $30000 \mathrm{~K}$ no helium-poor stars were found, but two stars in OriOBI $I_{\mathrm{b}}$ are helium rich. These stars are $\sigma$ OriE and HD 37776. Both are found to be variable in I(4026), and for $\sigma$ OriE Thomsen (1974) has found the variations to be periodic.

The spectral range B0-B2 is particularly suitable in deriving the helium abundances, because the model-atmosphere computations have revealed that a linear combination of $I(4026)$ and the $H \beta$ index, that is observed by D. Crawford and collaborators, is nearly independent of effective temperature and surface gravity, but a sensitive function of the helium-to-hydrogen ratio. This means that differential helium abundances can be directly determined from the observed values of $I(4026)$ and $\mathrm{H} \beta$, both of which indices are independent of interstellar reddening.

The mean helium abundances for different groups of stars as derived from stars in the spectral range B0-B2 are given in Table $\mathrm{I} . N$ is the number of stars in each group, and $\langle y\rangle$ is the mean logarithmic helium-to-hydrogen ratio. The $\langle y\rangle$ value for the field stars is normalized to -1.00 , and the quoted errors, that are derived from the rms scatter of the individual $y$ values, refer to differential helium abundances. From Table I I conclude that two groups of stars, namely $h+\chi$ Per and CepOBIII, have an apparently lower helium abundance than the rest of the stars observed.

Details of this work will be given elsewhere.

TABLE I

\begin{tabular}{lrll}
\hline Group & $N$ & $\langle\epsilon(\mathrm{He})\rangle$ & $\langle y\rangle \pm \sigma(\langle y\rangle)$ \\
\hline Field stars & 33 & 0.100 & -1.00 \\
Sco-Cen & 12 & 0.101 & $-0.99 \pm 0.02$ \\
NGC 6231 & 10 & 0.087 & $-1.06 \pm 0.03$ \\
$h+\chi$ Per & 12 & 0.059 & $-1.23 \pm 0.03$ \\
CepOBIII & 10 & 0.060 & $-1.22 \pm 0.03$ \\
LacOBI $_{\text {OriOBI }}$ & 6 & 0.085 & $-1.07 \pm 0.03$ \\
\hline
\end{tabular}




\section{References}

Garrison, R. F.: 1967, Astrophys. J. 147, 1003.

Nissen, P. E.: 1974, Astron. Astrophys. 36, 57.

Strömgren, B.: 1966, Ann. Rev. Astron. Astrophys. 4, 433.

Thomsen, B.: 1974, Astron. Astrophys. 35, 479.

\section{DISCUSSION}

Spinrad: Please elaborate on the galactocentric distances of the clusters with various He abundances, which you have just described.

Nissen: The associations with a helium-to-hydrogen ratio of $N_{\mathrm{He}} / N_{\mathrm{H}}=0.10$, namely Sco-Cen, Ori $\mathrm{OBI}_{b}$, Lac OBI $\mathrm{O}_{b}$, all lie within a distance of $600 \mathrm{pc}$ from the Sun. The NGC 6231 cluster, for which $N_{\mathrm{He}} / N_{\mathrm{H}} \cong 0.10$, lies in the Sagittarius spiral arm, $2000 \mathrm{pc}$ away from the Sun. The $h+\chi$ Per cluster with the low helium abundance of $N_{\mathrm{He}} / N_{\mathrm{H}}=0.06$ lies in the outer spiral arm at a distance of $2000 \mathrm{pc}$. Finally CepOBIII, for which $N_{\mathrm{He}} / N_{\mathrm{H}}=0.06$ lies at a distance of $800 \mathrm{pc}$ in nearly the same direction as $h+\mathrm{x}$ Per.

Stephenson: Did you say anything about the non-influence of stellar rotation on your 4026 index, and if so would you please repeat what it was you said?

Nissen: No, I did not say anything about the influence of stellar rotation on I(4026). However, in an earlier publication (Astron. Astrophys. $36(1974), 57)$ I investigated if there was any dependence of the derived helium-to-hydrogen ratios of field stars on rotational velocity and I did not find any effect for projected rotational velocities below $300 \mathrm{~km} \mathrm{~s}^{-1}$.

Morgan: There is a large systematic error in the Henry Draper Catalogue for faint $B$ stars in the Orion region. Stars having HD types of B8 and B9 have MK types of around B3 to B5.

Nissen: This means that one needs MK types in order to see if there is a discrepancy between colours and spectral types for the stars in Orion, which I find to be apparently helium poor.

Walborn: (1) Can you say anything about periodicities in $\sigma$ Ori $\mathrm{E}$ in your own $\lambda 4026$ data?

(2) How large a telescope would be needed to reach stars in the Magellanic Clouds $\left(\sim 16^{m}\right)$ with your system?

Nissen: (1) No, I can only refer to B. Thomsen's observations.

(2) With a 4-m telescope it should be possible to determine the $I(4026)$ index for the early B-type stars with an accuracy of $0 . m^{m} 05$ if several hours of integration time per star is applied. 